\title{
6 Ungeeignete Wundtherapeutika
}

Aus früheren Zeiten und aus „Hausmitteln“ verschiedener Länder halten sich Lokaltherapeutika und Methoden, die in der modernen Wundbehandlung nicht angebracht sind. Früher waren auch in der Medizin Produkte im Einsatz, die sich hartnäckig halten. Schädigende/allergene Wirkungen der Inhaltsstoffe waren z. T. noch nicht bekannt.

In der Therapiefindung muss der aktuelle Stand der Wissenschaft um die Wundursache und die physiologischen Vorgänge bei der Wundheilung berücksichtigt werden. „Ich mache das schon seit Jahrzehnten so“ oder ähnliche Ausführungen sind keine Begründungen oder Freibriefe für die Behandlung mit den nachfolgend aufgeführten Therapeutika.

\subsection{Lokalantibiotika}

Beispiele: Framycetin (Leukase), Gentamycin, Metronidazol, Neomycin, Tetrazykline.

Diese Stoffe haben nur eine oberflächliche Wirkung. In tieferen Gewebeschichten erreichen sie keinen Wirkspiegel. Nur eine systemische Antibiotikatherapie erreicht alle Bakterien in Wunden. Durch die oberflächliche Anwendung besteht die Gefahr der Züchtung resistenter Bakterienstämme in der Wunde und die Entstehung eines allergischen Kontaktekzems ist möglich.

\section{Ausnahme in der Palliativmedizin}

Metronidazol als $1 \%$ steriles Gel bei übelriechenden malignen Wunden.

\subsection{Farbstofflösungen}

Beispiele: Brillantgrün, Eosin, Kristallviolett, Methylviolett, Pyoktanin.

Diese Produkte machen durch Verfärbungen eine Beschreibung der Wunde bei der Wundinspektion unmöglich. Die Substanzen weisen große Wirkungslücken mit schwacher antibakterieller und antimykotischer Wirkung auf. Oft trocknen sie die Wunde aus und sind massiv zelltoxisch und schwermetallbelastet. 


\subsection{Veraltete Antiseptika}

Beispiele: Chlorhexidin Lsg. 0,2\%, Ethacridinlactat (Rivanol), Kaliumpermanganat, Wasserstoffperoxid $3 \%$.

Veraltete Antiseptika haben oft eine unzureichende antiseptische Wirkung. Sie sind gewebereizend, gewebetoxisch und hemmen die Wundheilung. Eine ordentliche Wundinspektion ist durch Verfärbungen nicht möglich. Außerdem verfärben sie Wäsche irreversibel.

\subsection{Polyvidon-Jod-Lösung/Jodoformgaze}

Beispiele: Betaisadona, Opraclean.

- Jod wirkt antiseptisch mit breitem Wirkspektrum.

- Brennt beim Einbringen in die Wunde.

- Wird durch Blut, Eiter und Wundexsudat inaktiviert (sogenannter „Eiweißfehler").

- Bei langdauernder Anwendung mögliche negative Wirkung auf die Wundheilung.

- Gefahr des Auftretens von kontaktallergischen Reaktionen durch den Kontakt mit dem Jodkomplex.

- Die Anwendung als Salbe ist wegen des Exsudatstaus ungünstig.

- Durch Jodaufnahme und Jodüberangebot kann eine Hyperthyreose bis zur lebensbedrohlichen jodinduzierten thyreotoxischen Krise ausgelöst werden.

\section{Kontraindikationen}

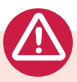

Stillzeit, Jodallergie, Dermatitis herpetiformis, Radiojodtherapie, Acne vulgaris.

Die Indikationen von Präparationen mit PVP-Jod in der Wundbehandlung sind in erster Linie akute Wunden. In der Behandlung von Patienten mit chronischen Wunden ist PVP-Jod entbehrlich. Spülungen von Wund- und Körperhöhlen mit PVP-Jod oder dessen Instillation sind wegen der Gefahr einer erhöhten Jodaufnahme zu meiden.

Alternativen: Antiseptika wie Lavasept-Lösung, Lavanox oder Octenisept.

Zur Behandlung von Abszesshöhlen und gespaltenen Fisteln werden sterile Gazetamponaden verwendet. Diese können mit Lavasept-Lösung getränkt sein, hydrophob sein (CutimedSorbact) oder mit Polyhexanid-Macrogolsalbe versetzt werden (Tamponadefunktion/Blutstillung, Wunddesinfektion). 


\subsection{Weitere ungeeignete Produkte}

Dies sind oft Produkte aus anderen Anwendungsbereichen ohne Eignung oder Prüfung. Solche Produkte sind für Patienten als „gefährlich“ zu werten und daher nicht indiziert.

- Salben: verhindern den Abfluss von Wundexsudat und bilden eine feuchte Kammer

- Puder: trocknen die Wunde aus, verklumpen und behindern die Granulation

- Lebensmittel, z. B.: Speisehonig, Rohrzucker, Salz, Quark, rohe Eier, Kohlblätter, Ochsenblut, Walnussblätterbrei, Rotwein, Pulverkaffee,Teebeutel, Zitronensaft;

Produkte haben keine Zulassung zur Wundtherapie $\rightarrow$ Ausnahme: Honig als Medizinprodukt

- Haushaltsmittel, z. B.: Zeitungspapier, Sand, Zahnpasta, Heilerde, Benzin, Glycerin, Teebaumöl, Lavendelöl, Waffenöl (Ballistol); Produkte haben keine Zulassung zur Wundtherapie

- Alkoholische Lösungen: sind schmerzhaft und zytotoxisch

- Off-Label-Use von Arzneimitteln, z. B. Insulin, Heparin, Vitamine, Glukoseinfusionslösungen: Medikamente ohne Zulassung für die Wundtherapie, daher haftungsrechtliche Probleme; sie gelten oft „Geheimtipp“ jedoch ohne wissenschaftliche Evidenz.

\subsection{Nicht geeignete Methoden und Vorgehensweisen}

Auskühlen der Wunde: Wenn eine Wunde abkühlt, dann laufen alle Reparaturvorgänge langsamer ab, da alle biochemischen Vorgänge temperaturabhängig sind. Unter $28^{\circ} \mathrm{C}$ findet keine Phagozytose oder Zellteilung statt. Somit ist die Behandlung, bei der die Wunde „gelüftet“ wird, obsolet. Spüllösungen sollen aus diesem Grund körperwarm angewendet werden.

Anwendung enzymatischer Präparate auf trockenen Nekrosen: Die Enzyme wirken nur in feuchten Wunden.

Trockene Wundbehandlung: Diese Wundbehandlungen sind schmerzhaft, trocknen an, kühlen die Wunde aus und verursachen beim Entfernen Schmerzen. Die physiologischen Vorgänge der Wundheilung benötigen ein feuchtes Wundmilieu.

Einen Ausnahmefall stellen Wunden bei pAVK dar. Hier kommen nur trockene Verbände als Infektionsprophylaxe in Frage. 


\section{Ungeeignete Wundtherapeutika}

Wundbäder: Das Baden von Wunden gilt als unzeitgemäß. Es werden Wachstumsfaktoren und Zytokine ausgeschwemmt. Weiterhin besteht die Problematik der Keimverschleppung. Oft besteht mangelnder Kontakt zum Wirkstoff und die Gefahr der falschen Wirkstoffkonzentration sowie die Infektgefahr durch unsterile Zubereitung bei der Herstellung.

Tägliche Inspektion der Wunde: Das Öffnen des Verbands in der Granulationsund Epithelisierungsphase ist kontraproduktiv, da das Einhalten einer möglichst langen Wundruhe sehr wichtig ist. Sitz und Beschaffenheit der Wundauflage selbst müssen trotzdem möglichst täglich kontrolliert werden und z.B. durchnässte Verbände ausgetauscht werden. 